\title{
Is There a Specific Hemodynamic Effect in Reflexology? A Systematic Review of Randomized Controlled Trials
}

\author{
Jenny Jones, BSc (Hons), RN, Patricia Thomson, PhD, MPH, MA, DipN, RN, RNT,2 \\ Kathleen Irvine, BSc (Hons), Dip. Lib, Dip Ed. Tech, MCLIP, \\ and Stephen J. Leslie, BSc, MB, ChB, FRCP, PhD ${ }^{1,4}$
}

\begin{abstract}
Objectives: Reflexology claims that the feet are representative of the body and that massage to specific points of the feet increases blood supply to "mapped" organs in the body. This review provides the first systematic evaluation of existing reflexology randomized controlled trials (RCTs) to determine whether there is any evidence to suggest the existence of a reflexology treatment-related hemodynamic effect; to examine whether reflexology researchers used study designs that systematically controlled for nonspecific effects in order to isolate this specific component; and to highlight some of the methodological challenges that need to be overcome to demonstrate specific and beneficial hemodynamic effects.

Design: Fifty-two RCTs of reflexology published from 1990 to September 2011 were initially retrieved.

Setting/Location: Cardiorespiratory Department, Highland Heartbeat Centre, Raigmore Hospital, Inverness. Subjects: Adult subjects.

Interventions: Studies using reflexology foot massage techniques as the intervention versus sham reflexology treatment, simple foot massage, conventional treatment, or no treatment as the control were then selected.

Outcome measures: Outcome measures included any hemodynamic parameter potentially involved in the regulation of circulating blood volume and flow, including heart rate and systolic and diastolic arterial blood pressure. Results: Seven RCTs suggested that reflexology has an effect on selected cardiovascular parameters; however, five of these delivered the reflexology intervention as a whole complex treatment, with the data collector often delivering the intervention themselves.

Conclusions: This systematic review found that although reflexology has been shown to have an effect on selected hemodynamic variables, the lack of methodological control for nonspecific general massage effects means that there is little convincing evidence at this time to suggest the existence of a specific treatment-related hemodynamic effect. Furthermore, the review found that few studies of reflexology controlled for nonspecific effects in order to isolate any specific active component, despite the hemodynamic claim being a key part of the therapeutic value of reflexology. Therefore, further research approaches using more innovative designs and robust methods that can allow a treatment-induced, therapeutically beneficial hemodynamic effect to reveal itself are needed to help reflexology purchasers make a more informed decision about the safety and product quality of the reflexology hemodynamic claim and for reflexologists to be able to guarantee minimum product quality, validity, and safety standards in their practice.
\end{abstract}

\section{Introduction}

A NNUAL EXPENDITURE ON COMPLEMENTARY and alternative medicine (CAM) therapies in the United Kingdom is estimated to be in excess of $£ 1.6$ billion per annum. Reflexol- ogy is one of the most popular forms of complementary therapy massage in the UK. ${ }^{1,2}$ The theory of reflexology is based on the idea that the feet represent a scaled and proportioned map of the human body, with discrete areas of the feet (found mostly on the planter tissue region) being

\footnotetext{
${ }^{1}$ School of Nursing, Midwifery \& Health, University of Stirling, Highland Campus, Inverness, United Kingdom.

${ }^{2}$ School of Nursing, Midwifery \& Health, University of Stirling, Stirling Campus, Stirling, United Kingdom.

${ }^{3}$ Highland Health Sciences Library, Centre for Health Science, Inverness, United Kingdom.

${ }^{4}$ Highland Heartbeat Centre, Cardiology Unit, Raigmore Hospital, Inverness, United Kingdom.
} 
associated with individual organs of the body. ${ }^{3}$ Many reflexologists believe that the application of massage pressure to these areas, using touch techniques unique to reflexology, stimulates an increase in blood supply to the corresponding organ associated with the foot area being massaged. Furthermore, this hemodynamic outcome is a specific effect inherent in reflexology that is distinct from ordinary foot massage. $^{3-6}$ This hemodynamic treatment-related effect is believed to be consistently therapeutically beneficial. ${ }^{7}$

Given the public-driven financial investment in this therapy, reflexology safety and product quality has become a CAM health care research priority, ${ }^{8}$ particularly since reflexology, unlike any other CAM therapy, makes unique claims for a specific, therapeutically beneficial hemodynamic effect. Since it is this effect that distinguishes reflexology from simple foot massage, it is a core part of its inherent value. Therefore, when a therapy makes such a unique prediction as this, rigorous evidence is needed to determine that the practice delivers the product quality that it claims to and that its specific effects are safe and effective for all users, ${ }^{8}$ particularly any relevant patient subgroups that may be at risk from unanticipated adverse treatment-related reactions. ${ }^{9}$

At this time, four systematic reviews of reflexology efficacy have been published and none have found enough evidence to support the use of reflexology for the majority of human conditions. ${ }^{1,2,10,11}$ However, the scope of these reviews did not extend beyond overall treatment evaluation for a variety of human conditions. None of the articles referred to or examined the claim for a specific hemodynamic effect or identified any research that had isolated a specific hemodynamic component or discussed the potential challenges that reflexology researchers face in attempting to undertake such an enquiry. This could mean that existing reflexology research may already offer evidence for a specific hemodynamic effect as claimed, even if the overall study outcomes were not significant, and researchers may have already used novel methods to isolate such an effect even though the overall trial outcomes were negative. To date, however, the reflexology evidence base has not been searched with these criteria in mind.

Since the reflexology profession now aims to meet the minimum quality and safety standards set down by the Complementary and Natural Healthcare Council (CNHC) in order to join its voluntary UK CAM therapist register and integrate more with evidence-based conventional medicine, the question of identifying evidence to support the claims of a specific hemodynamic effect has become an urgent priority with regard to quality and safety. If reflexologists continue to make unique claims for specific effects beyond those of a general foot massage, these specific effects must be considered to be the most valuable therapeutic components the therapy can produce. If the claims are to be upheld in reflexology training literature and advertising, like all public healthcare interventions, it must be supported by evidence.

With this requirement in mind, the aim of this review was to provide the first systematic evaluation of existing randomized controlled trials (RCTs) of reflexology to identify whether there is any evidence to suggest a reflexology treatment-related, specific hemodynamic effect on individual organ perfusion levels, which, regardless of the organ involved, should be differential to a generalized hemodynamic response to nonspecific effects. A further aim was to identify whether reflexology researchers have used study designs that systematically controlled for nonspecific effects in order to isolate this specific active component. The final aim was to highlight some of the methodological challenges that reflexology researchers need to overcome in order to demonstrate a specific, safe, and therapeutically beneficial hemodynamic effect as claimed.

\section{Methods \\ Eligibility criteria}

Due to the heterogeneity of published reflexology studies, titles and abstracts of RCTs of reflexology using adult subjects (including healthy volunteers) without age limitation and including physiological outcomes for any type of human condition, published from 1990 to September 2011, were initially retrieved. No language limits were applied. Although there has been criticisms about the assumptions of placebo-controlled RCTs being biased in favor of specific effects more often found in drug trials, rather than the complex array of both specific and nonspecific effects found in CAM therapies, ${ }^{12}$ the RCT methodology inclusion criteria was applied. This seemed appropriate as the review concerns reflexologists' claims of specific effects. The RCT design is thought to be the most reliable form of primary health care evidence for evaluating complex interventions such as reflexology when a relevant systematic review of RCTs is not available. $^{13,14}$

\section{Types of interventions and controls}

RCTs featured reflexology foot massage techniques as the intervention versus sham reflexology treatment, simple foot massage, conventional treatment, or no treatment as the control. There was no restriction on the duration or style of reflexology treatment, or when or where it was applied.

\section{Information sources and search strategy}

We searched MEDLINE, CINAHL, EMBASE, British Nursing Index, and the Cochrane Database of Systematic Reviews. The Association of Reflexologists (the United Kingdom's largest professional register of reflexology therapists) was contacted, and its reference list of reflexology articles and studies was reviewed. The bibliographic references from all retrieved articles were also manually searched in order to find studies not identified by electronic searches. See Table 1 for the database search strategy.

\section{Study selection}

The titles and abstracts of all identified RCTs involving any type of reflexology and using adult subjects were read to determine eligibility. Relevant full-text articles were retrieved (Fig. 1). After applying the inclusion and exclusion criteria, articles that included primary or secondary hemodynamic outcomes were identified. Studies using foot massage rather than reflexology therapy were excluded even if they included hemodynamic outcomes.

\section{Objective outcome measures}

From these articles, the primary outcomes were whether and to what extent there was any scientific evidence to 
Table 1. Database Search Strategy

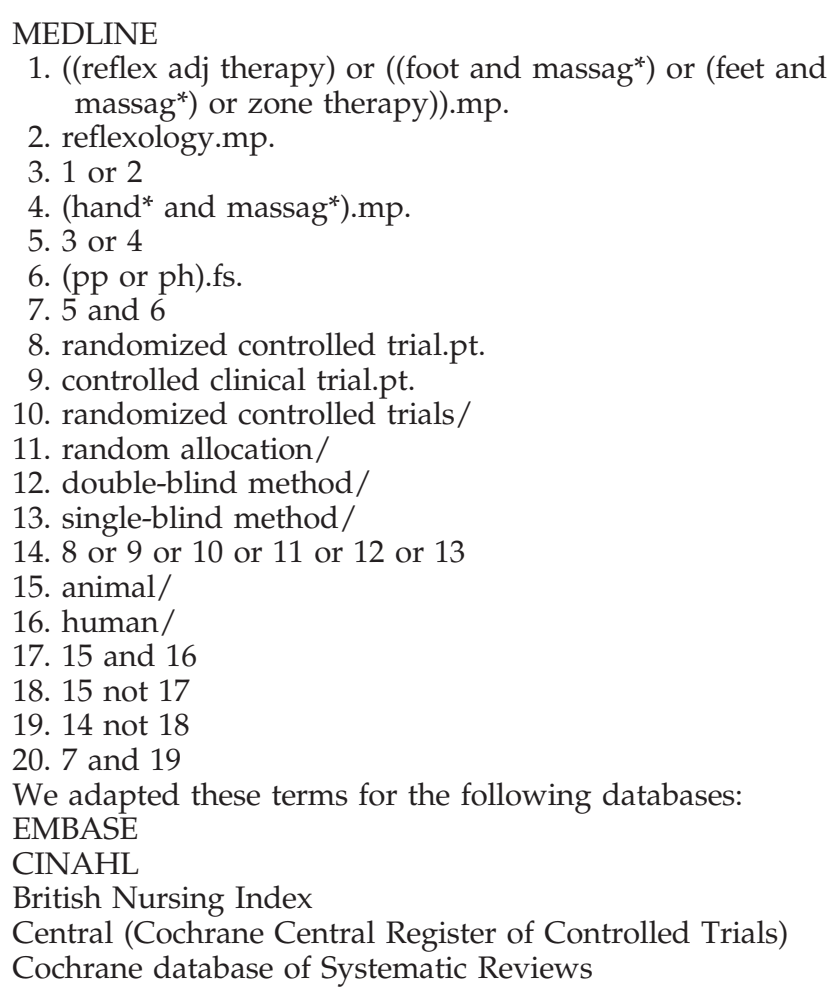

suggest that reflexology treatment to distinct areas of the feet (reflex points), using touch techniques unique to reflexology, produces a specific and therapeutically beneficial hemodynamic effect, from which it can be inferred that an increase in blood supply to the corresponding organ or organs occurred as claimed.

\section{Data items}

Outcomes included any hemodynamic parameter potentially involved in the regulation of circulating blood volume and flow, including heart rate and systolic and diastolic arterial blood pressure. These were selected because they have a common mean metric across all the studies and could be used to infer a change in organ perfusion rates. Other less common parameters were included if directly relevant to the enquiry.

\section{Risk of bias in individual studies}

The methodological quality of identified articles was assessed by JJ and KI using The Cochrane Library recommended Quality Assessment Tool for Quantitative Studies checklist (EPHPP). ${ }^{15} \mathrm{JJ}$ and KI independently scored each identified article based on the tool criteria. Selection bias, study design, confounders, blinding, data collection methods, withdrawals and drop-outs, intervention integrity, and analysis components of each study were rated. Scores in the checklist categories summary range from 1 to 3 , with 1 indicating the highest strength. Both reviewers then scored each article overall as either $1=$ strong, $2=$ moderate, or $3=$ weak using the EPHPP global criteria and assessed the quality of the identified studies independently. It was plan-

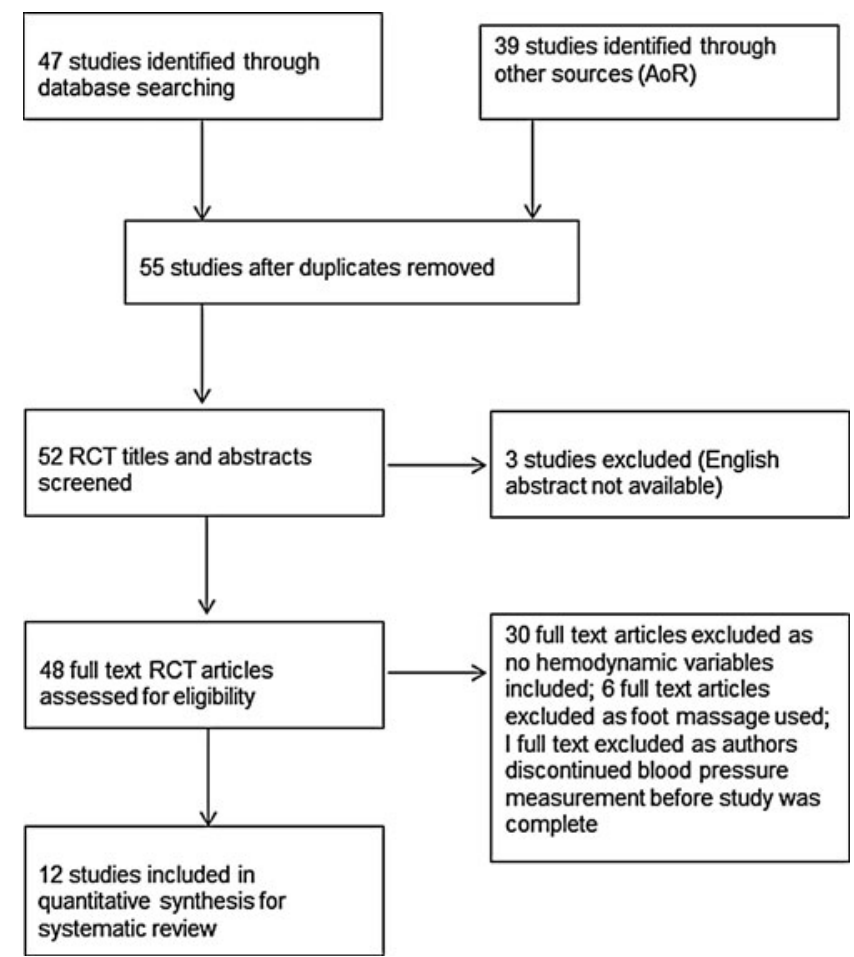

FIG. 1. PRISMA flow diagram. Study selection and review process.

ned that any disagreement would be resolved by discussion and consensus with SJL; however, both reviewers agreed on all global scores.

The studies were assessed as being double-blinded if the participants, data collector, and/or data analyst were blinded to intervention allocation since therapist blinding is not possible in the special case of reflexology. Otherwise, the trial was defined as a controlled clinical trial. Systematic differences in the care delivered to study participants, which can introduce performance bias, were accounted for by our explicit inclusion and exclusion criteria being applied to the studies under consideration. ${ }^{16}$

\section{Results}

Out of the 48 RCT full-text reflexology research articles retrieved, 12 articles that included primary or secondary hemodynamic outcomes were identified. ${ }^{17-26}$ Five RCT studies using foot massage rather than reflexology therapy ${ }^{27-31}$ were excluded even though they included hemodynamic outcomes. The 12 RCTs involving a reflexology intervention met with our inclusion criteria and contained sufficient and appropriate hemodynamic data. Their key data are summarized in Table 2. The experimental design quality of the studies was assessed using the Critical Appraisal Skills Program (CASP) critical analysis tool. ${ }^{32}$ Although the majority of studies used common hemodynamic outcomes, the lack of understanding of how reflexology works made classification of the interventions difficult. ${ }^{14}$ The analysis showed that there was significant heterogeneity; therefore, systematic meta-analysis of the data was not possible. So for this systematic review, the studies were differentiated first by EPHPP score, then by type and duration of reflexology 


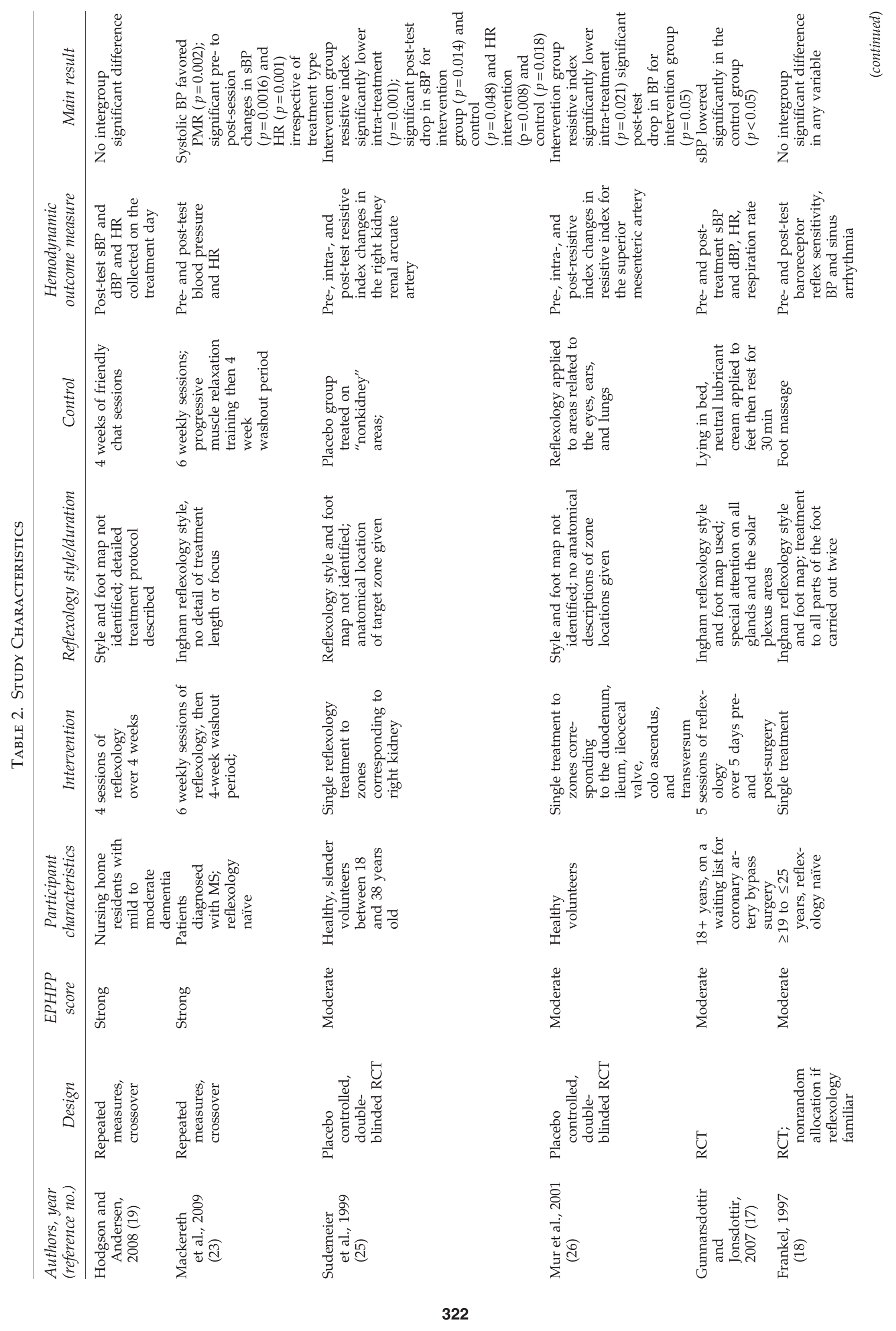




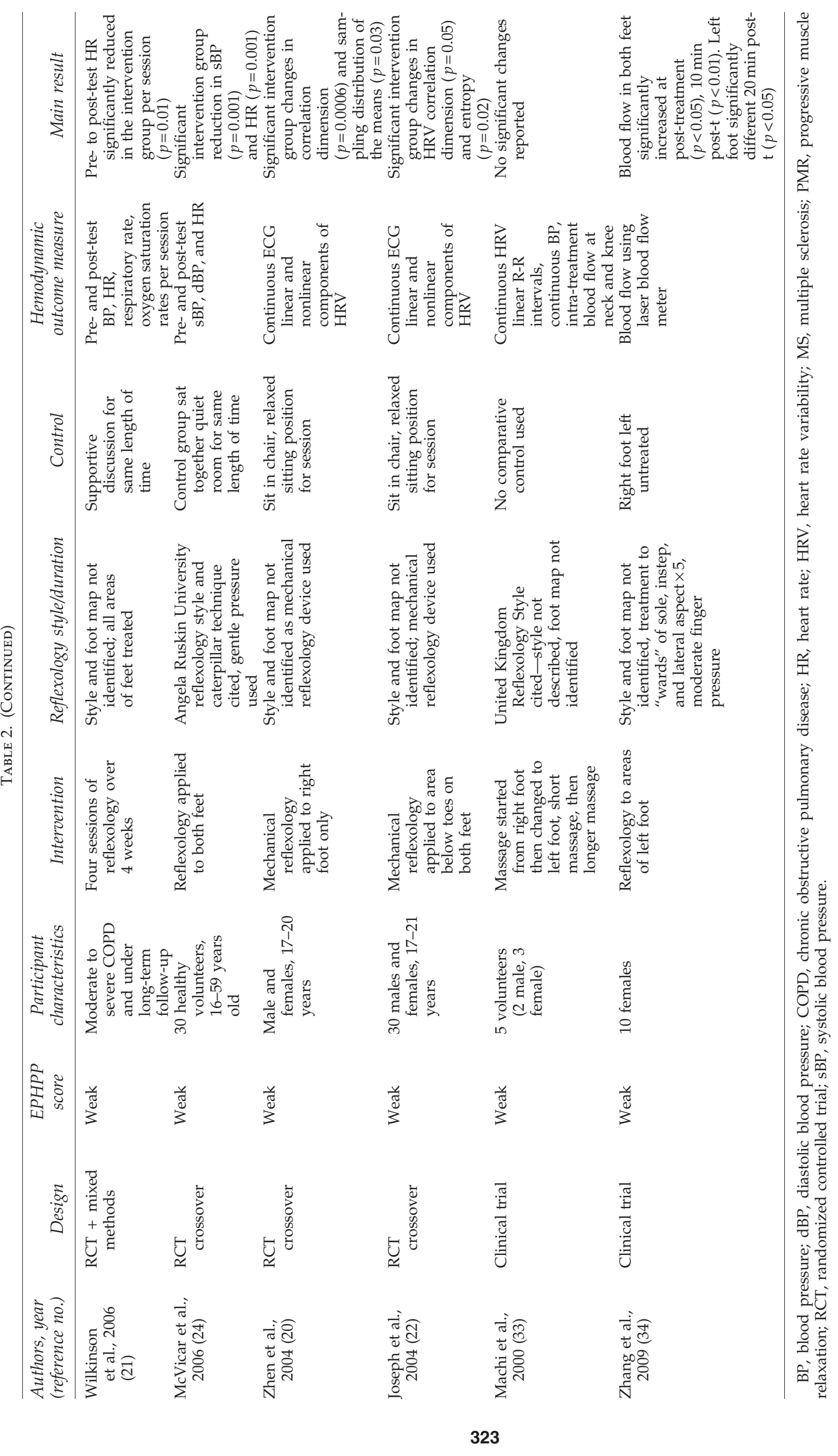


intervention, and finally by common and less common interpretable hemodynamic parameters.

\section{EPHPP scores}

Differentiating the studies by EPHPP quality score (Table $3)$, six had a global rating of "weak,"20-22,24,33,34 four were rated as "moderate"17,18,25,26 and two scored as "strong". 19,23

\section{Type and duration of reflexology intervention}

Reflexologists learn the location of each body part or organ thought to be reflected on the feet by studying reflexology foot maps. Reflexology technique and foot maps can vary considerably, dependent on where and with whom the reflexologist trained. Three articles quoted the Ingham method as the reflexology intervention style used. ${ }^{17,18,23}$ The Ingham style has its own standardized technique and foot map and is taught by the British School of Reflexology, one of the largest UK reflexology teaching providers. ${ }^{35}$ One article specified Angela Ruskin University reflexology as the intervention type, ${ }^{24}$ and one described the therapy style as United Kingdom style reflexology. ${ }^{33}$ Neither of these articles described how these techniques differentiated from other forms of reflexology, nor indicated whether each had its own individualized foot map. Of the remaining articles, seven gave no indication of type of reflexology style or variation of foot map used. ${ }^{19-22,25,26,34}$ Only one specified the exact anatomical location for both the intervention and control areas of the feet, ${ }^{25}$ and only one provided a detailed protocol sequence for the reflexology intervention. ${ }^{19}$

\section{Methodological approaches to isolating specific versus nonspecific effects}

Ten of the 12 studies delivered the reflexology intervention as a "whole system" complex treatment, making no attempt to control for nonspecific hemodynamic effects in order to isolate any active specific hemodynamic ingredient said to be inherent in the therapy. Control comparisons included "friendly chat sessions"19 or "symptom discussion" sessions for the same duration as the reflexology intervention, ${ }^{21}$ progressive muscle relaxation training, ${ }^{23}$ foot massage, ${ }^{18}$ sitting in quiet room, ${ }^{24}$ sitting in a relaxing chair, ${ }^{20,22}$ and resting in bed. ${ }^{17}$ Only two studies attempted to isolate a specific hemodynamic effect by contrasting massage to a specific area of the foot whilst measuring the associated organ perfusion rates compared with massage to "non-active" areas of the feet as the control. ${ }^{25,26}$

\section{Dosage}

Reflexology "dosage" appeared to differ considerably. Five studies used a single reflexology treatment as the intervention. $^{18,20,22,24,34}$ Four used a series of reflexology treatment sessions to form a combined measurable intervention. ${ }^{17,19,21,23}$ One study used both a single visit and an aggregated series of treatments as two separate measurement points. ${ }^{23}$ Two used a mechanical reflexology device ("Massager-Scroller") to massage a single area of the foot only. ${ }^{20,22}$

\section{Duration}

The length of the intervention also varied considerably, ranging between 8-minute treatment, ${ }^{25} 10$ minutes, ${ }^{34}$ "a short massage (10 minutes) and a long massage (20 minutes), ${ }^{\prime 33} 20$ minutes, ${ }^{20} 30$ minutes, ${ }^{19} 40$ minutes, ${ }^{23} 45$ minutes, $^{18} 50$ minutes, ${ }^{21}$ and 60 minutes, ${ }^{24}$ with no clear rationale offered in any article as to how the treatment length was determined.

\section{Common hemodynamic parameters}

Blood pressure. Two studies found significant post-test reductions for the reflexology intervention group in systolic blood pressure but not for diastolic blood pressure $(p=0.001)^{24}$ and $(p=0.05) .{ }^{26}$ One study found post-test significant reductions in systolic blood pressure in both the intervention group $(p=0.014)$ and control group $(p=0.048),{ }^{25}$ and another found a significant post-test difference irrespective of treatment type $(p=0.0016) .{ }^{23}$ Four studies failed to show any significant hemodynamic effect pre- to posttreatment on systolic or diastolic blood pressure arising from a reflexology intervention. ${ }^{18,19,21,33}$

Heart rate. One study found significant post-test heart rate reductions for both the treated $(p=0.008)$ and control group $(p=0.018) .{ }^{25} \mathrm{~A}$ different one reported significant post-

Table 3. EPHPP Methodological Quality Review Scores

\begin{tabular}{|c|c|c|c|c|c|c|c|}
\hline Study & $\begin{array}{l}\text { Selection } \\
\quad \text { bias }\end{array}$ & $\begin{array}{l}\text { Study } \\
\text { design }\end{array}$ & Confounders & Blinding & $\begin{array}{l}\text { Data collection } \\
\text { methods }\end{array}$ & $\begin{array}{l}\text { Withdrawals } \\
\text { and dropouts }\end{array}$ & $\begin{array}{l}\text { Global } \\
\text { rating }\end{array}$ \\
\hline Machi et al. ${ }^{33}$ & Weak & Weak & Weak & Weak & Strong & Weak & Weak \\
\hline Wilkinson et al. ${ }^{21}$ & Moderate & Moderate & Weak & Weak & Moderate & Moderate & Weak \\
\hline Joseph et al. ${ }^{22}$ & Weak & Weak & Weak & Weak & Weak & Weak & Weak \\
\hline Zhang et al. ${ }^{34}$ & Weak & Weak & Weak & Weak & Strong & Moderate & Weak \\
\hline McVicar et al. ${ }^{24}$ & Moderate & Strong & Weak & Moderate & Strong & Weak & Weak \\
\hline Zhen et al. ${ }^{20}$ & Weak & Strong & Weak & Weak & Strong & Moderate & Weak \\
\hline Sudemeier et al. ${ }^{25}$ & Weak & Moderate & Strong & Moderate & Strong & Strong & Moderate \\
\hline Frankel. $^{18}$ & Weak & Strong & Strong & Moderate & Strong & Strong & Moderate \\
\hline Mur et al. ${ }^{26}$ & Weak & Strong & Strong & Moderate & Strong & Moderate & Moderate \\
\hline Gunnarsdottir and Jonsdottir ${ }^{17}$ & Moderate & Strong & Strong & Weak & Strong & Strong & Moderate \\
\hline Hodgson and Anderson ${ }^{19}$ & Moderate & Moderate & Strong & Moderate & Strong & Strong & Strong \\
\hline Mackereth et al. ${ }^{23}$ & Moderate & Strong & Strong & Moderate & Strong & Strong & Strong \\
\hline
\end{tabular}


test heart rate changes for the intervention group irrespective of single reflexology treatment versus series of treatments intervention $(p=0.01) .{ }^{23}$ Still another study found significant post-test heart rate reduction for the intervention group $(p=0.01){ }^{21}$ Three studies failed to show any specific effect on heart rate for the intervention groups. ${ }^{17,19,26}$

\section{Other hemodynamic parameters}

Heart rate variability. Two nonlinear heart rate variability (HRV) parameters, the estimate of the standard deviation of the sampling distribution of the means (SENN) of R-R intervals and correlational dimension (CD) analysis, were found to be significantly different (SENN, $p=0.03$; CD, $p=0.0006$ ) in favor of the reflexology group in comparison to a control group. ${ }^{20}$ One study found significant pre- to postintervention group changes in HRV entropy, which is a thermodynamic quantity describing the amount of disorder in a system $(p=0.025) .^{22}$

Baroreceptor reflex sensitivity and sinus arrhythmia. Analysis of baroreceptor reflex sensitivity data and sinus arrhythmia rates revealed no significant difference when a reflexology intervention group was compared with a foot control massage group. ${ }^{18}$

Blood flow rates. Analysis of post-test pedal blood flow recovery rates found a significant 20 -minute post-treatment difference for the intervention group. ${ }^{34}$ Two studies attempted to measure the relationship between massage to specific areas of the feet and changes in localized vascular resistive index rates using color Doppler sonography. Both these studies reported positive findings for the intervention treatment, one reporting a significant intratreatment change in resistive index of the renal artery when the "kidney" are of the foot was massage $(p \leq 0.001) .^{25}$ The other found significant resistive index changes in the superior mesenteric artery $(p=0.021)$ when the "intestinal" reflex point on the foot was stimulated. ${ }^{26}$

\section{Study selection, eligibility, characteristics, and results:}

A summary of study characteristics is provided in Table 2.

\section{Risk of bias across studies}

The majority of reflexology RCTs appear to lack sufficient blinding, either because the data collector seemed to be aware of the group conditions during the study $20,21,23,33,34$ or because the data collector delivered the intervention reflexology itself. ${ }^{17,18,24}$ This can, in some instances, lead to selective reporting within the study and potentially affect the cumulative evidence. ${ }^{36}$ Only two studies were double-blinded, designed so that neither the data collector nor the examined subjects were aware of which group they belonged to. ${ }^{25,26}$

\section{Discussion}

This is the first systematic review of existing RCTs of reflexology to identify whether there is any evidence to suggest that massage to distinct areas of the feet (reflex points) using touch techniques unique to reflexology, produces a treatment-related hemodynamic change in individual organ perfusion levels that is quite distinct from nonspecific sys- temic hemodynamic effects. Furthermore, it is the first article to systematically review how reflexology researchers deliver the complex reflexology intervention and to determine if studies isolate this specific effect from within a complex multicomponent intervention in order to provide evidence to support its existence. This review is also unique in that it has attempted to highlight some of the methodological challenges that reflexology researchers need to overcome in order to demonstrate a specific and beneficial hemodynamic effect as claimed.

This review has shown that out of the 12 RCTs reviewed that used hemodynamic outcomes, there were seven whose findings suggest that reflexology massage has some kind of effect on blood pressure, heart rate, nonlinear components of $\mathrm{HRV}$, and vascular resistive index rates. Of these seven, two studies reported beneficial reductions in systolic blood pressure for the intervention group and two reported a reduction in the intervention groups mean heart rate. ${ }^{23-26}$ However, five of these seven studies reviewed delivered the reflexology intervention as a whole complex treatment, with the data collectors often delivering the intervention themselves. This meant that the study design was not able to provide an experimentally inert reflexology comparison that could differentiate between nonspecific hemodynamic effects such as the compassion of the therapist, treatment environment, the act of lying supine, and the effects of simple foot massage that would be equally present in both intervention and control. Therefore, the blood pressure and heart rate reductions are insufficient as evidence to suggest the existence of a specific change in the hemodynamic status of an individual organ at this time.

Overall, 10 of the 12 RCTs reviewed for this article did not systematically control for nonspecific effects in order to isolate any specific active component. The reasons for this are not clear. Yet as reflexologists make unique claims for specific effects beyond that of general foot massage, these specific effects must be considered to be the most valuable therapeutic components the therapy can produce. Therefore, if the claim is to be upheld in reflexology training literature and advertising, it must be supported, particularly because the reflexology profession now aims to achieve voluntary regulation through the $\mathrm{CNHC}$. Since the Department of Health recommends potential purchasers of CAM to consult a CNHC-registered practitioner whenever possible, the specific hemodynamic claim must be empirically measurable or the therapeutic claims modified to align with what can be experimentally demonstrated to be safe and valid. Reflexology is not unique in this requirement; evidence for safety and product quality and validity applies to all health care practices, regardless of origins.

To date, only two reflexology researchers have attempted to meet the design challenge regarding the distinction of specific versus nonspecific effects by using a double-blind randomized method purposefully designed to allow a specific effect to reveal itself. Intriguingly, both studies demonstrated what appeared to be a specific, hemodynamically beneficial effect on localized organ perfusion rates corresponding with reflexology massage to related areas of the feet, a hemodynamic effect that by virtue of their novel design strategy, appeared distinct from systemic nonspecific massage effects. ${ }^{25,26}$

Sudemeier et al. ${ }^{25}$ reported statistically significant changes in resistive index of the renal arcuate artery when the 
"kidney" foot area was massaged. Mur et al. ${ }^{26}$ found significant changes in the mesenteric artery resistive index when the "intestinal" area was massaged. ${ }^{26}$ The precise mechanism that caused the changes in resistive index in both intervention groups remains uncertain, although the direction of the change in resistive index allows the inference to be made that the change was therapeutically beneficial in both cases, as reflexologists claimed it would be. Furthermore, in both studies, no such change in resistive index occurred when control or unrelated points were massaged, implying that the effect was indeed specific as reflexologists claim. However, these two studies still demonstrate the challenges facing reflexology researchers with regard to measuring hemodynamic changes to specific organs without having to adopt invasive and potentially risky measuring methods. The researchers used noninvasive color Doppler sonography technology to infer arterial resistive index as an indication of organ blood flow velocity. This is certainly a move in the right direction, but the technology of color Doppler sonography has yet to be widely validated as a reliable method from which to infer perfusion flow rates. Furthermore, neither article defined the organ-associated foot reflexology map location used, which makes it difficult to reproduce their methods and findings even if the technology was more robust.

The scientific study of specific effects within reflexology is further complicated by the challenge of finding a suitable control that can allow any active ingredient in the intervention to reveal itself. In one recent systematic review of reflexology efficacy, ${ }^{2}$ the majority of studies ${ }^{19,37-45}$ used general foot massage as the experimental control. Yet in most cases, the study design is not clear on how the sham foot massage treatment can be differentiated from reflexology foot massage treatment. The researchers describe the sham treatment as either gentle foot massage or foot massage that simply avoids pressure on the reflex areas of the foot. ${ }^{19,41,43-46}$ However foot massage is too similar to reflexology foot massage treatment to be considered a distinct passive form of sham control.

Furthermore, this review has found that reflexology researchers do not appear to acknowledge the issue of reflexology foot chart inconsistency and the effect that this can have with regard to a rigorous standardization of the intervention and reproducibility of findings. ${ }^{47}$ Reflexologists learn the exact reflected location of each body part or organ on the feet by studying maps or charts. One of the most challenging aspects of reflexology research is that different schools of reflexology have since evolved with slightly varying charts. Some appear to have evolved from Traditional Chinese Medicine meridian maps, while other charts derived from the personal empirical or intuitive experiences of therapists or individual schools of reflexology. ${ }^{48}$

The lack of reflexology foot map standardization presents a serious research challenge in terms of how to tease out evidence of a specific hemodynamic effect on the internal organs quite distinct from the hemodynamic effects of conventional foot massage. After all, the principal claim of reflexology is the existence a two-way relationship between specific points or areas of the feet and the organs of the body that allows the emergence of a specific hemodynamic effect to be revealed at the opposite organ end. The vast majority of published reflexology studies do not give details of which reflexology map the intervention is founded upon, which means that some of the studies may be, in theory, using relatively different reflexology charts on which to base the specific reflex-point intervention. If this is the case, there is a lack of consistency in the active intervention approach, which arguably invalidates the validity and generalizability of research findings. This is a serious experimental challenge that reflexology researchers must overcome in order to produce results that are reliable and reproducible.

Other challenges include the question of how to isolate a specific reflexology effect without affecting its "potency" and how to measure an effect that uses unproven ideas of "energy" and no know means of a biological pathway. Furthermore, how to standardize the reflexology treatment within a controlled environment so as to control the "dosage" or active quantity. To meet these challenges and in the process, become more evidence-based, reflexology researchers must develop experimental protocol guidelines in order to establish whether more or longer reflexology sessions equate to a higher "dose," whether the specific hemodynamic effect is more "pressure" dependent than time dependent, and find the means and methods of standardizing and maintaining the dose potency.

Therefore, in order to rigorously evaluate the validity of reflexologists' claims of a specific hemodynamic component in reflexology, more innovative study designs are needed. These designs must allow for a specific, reflexology induced, and therapeutically beneficial hemodynamic effect to be able to demonstrate itself over and above the nonspecific effects involved in general massage-and meet the methodological standard of the double-blind RCT. In future, reflexology researchers need to develop a simple standardized reflexology intervention and a suitable form of control and identify clear and meaningful physiological outcome measures that can be objectively measured. They must ensure the "blindability" of the data collector and participants and overcome the issue of inconsistent reflexology foot maps. We have suggested one potential method elsewhere. ${ }^{49}$

\section{Limitations}

We did not systematically attempt to uncover unpublished studies, although searches of reference lists of published studies and systematic analysis did not identify unpublished material. Since some of the studies were carried out by reflexology therapists who measured the outcomes as well as delivering the treatment, this could lead to bias. Finally, the dose, duration, and type of reflexology varied considerably between studies, which could have influenced their findings.

\section{Conclusions}

This systematic review has provided the first evaluation of data from RCTs of reflexology to see whether there is any evidence to suggest the existence of a reflexology treatmentrelated hemodynamic change in individual organ perfusion levels that is quite distinct from nonspecific systemic hemodynamic effects. Furthermore, it is the first article to identify that few studies of reflexology controlled for nonspecific effects in order to isolate any specific active component, despite the hemodynamic claim being a key part of the therapeutic value of reflexology. The review also highlighted some of the methodological challenges evident in the existing reflexology evidence base that reflexology researchers need 
to overcome in order to demonstrate a specific and beneficial hemodynamic effect as claimed.

Therefore, further research approaches using more innovative designs and robust methods which can allow a treatment-induced, therapeutically beneficial hemodynamic effect to reveal itself are needed. Only by meeting these research challenges will reflexology researchers be able to provide high-quality evidence for users and purchasers of reflexology to help them make a more informed decision about the safety and product quality of the reflexology hemodynamic claim and enable reflexologists to guarantee minimum product quality, validity, and safety standards in their practice.

\section{Funding}

No external funding was provided for this review article.

\section{Acknowledgments}

The authors wish to thank the Association of Reflexologists.

\section{Author Disclosure Statement}

No competing financial interests exist.

\section{Contributors}

JJ and SJL conceived the systematic literature review hypothesis; JJ and KI created the literature search strategy and undertook the methodological assessment of retrieved literature; JJ wrote the first draft of the manuscript; and SJL, PT, and KI critically reviewed and contributed to the final draft. All authors are guarantors.

\section{Sponsorship}

This literature review was sponsored by the University of Stirling.

\section{Provenance and Peer Review}

Not commissioned; not externally peer reviewed.

\section{References}

1. Ernst E. Is reflexology an effective intervention? A systematic review of randomised controlled trials. Med J Aust 2009;191:263-265.

2. Ernst E, Posadzki P, Lee MS. Reflexology: An update of a systematic review of randomised clinical trials. Maturitas 2011;68:116-120.

3. International Institute of Reflexology. Original Inham Method Foot Wall Chart. 2011. http://store.reflexology-uk .net $/$ products?tag $=$ charts $\% 20 \% 26 \% 20$ promo Accessed November 01, 2011.

4. International Institute of Reflexology I. The nations leading authority. 2012. http://www.reflexology-usa.net/branches .htm Accessed January 30, 2011.

5. International Institute of Reflexology I. IIR Training: Why settle for less than the best? 2012. http://www.reflexology-uk .net/site/training-courses/iir-diploma-course Accessed January 07, 2011.

6. Ingham E. Reflexes Present in the Hands and Feet. Stories the Feet Can Tell Thru Reflexology; Stories the Feet Have
Told Thru Reflexology, 11th ed. St Petersburg, FL: Ingham Publishing; 1984:10-11.

7. Ingham E. The Original works of Eunice D Ingham; Stories the Feet Can Tell Thru Reflexology; Stories the Feet Have Told Thru Reflexology, 2nd ed. St Petersburg, FL: Ingham Publishing; 1992:3-4.

8. Robinson N, Lorenc A, Lewith G. Complementary and alternative medicine (CAM) professional practice and safety: A consensus building workshop. Eur J Integr Med 2011; 3:e49-e53.

9. Beckner W, Harlan W. The role of outcomes and observational research in evaluating integrative medicine. In: Clinical Research in Complementary Therapies: Principles, Problems and Solutions. Editor Lewith, G. 2nd ed. Edinburgh; Churchill Livingstone; 2012:61-72.

10. Botting D. Review of literature on the effectiveness of reflexology. Complement Ther Nurs Midwifery 1997;3:123-130.

11. Wang M, Tsai P, Lee P, Chang W, Yang C. The efficacy of reflexology: Systematic review. J Adv Nurs 2008;62:512-520.

12. Walach $\mathrm{H}$, Jonas WB, Lewith $\mathrm{G}$. The role of outcomes research in evaluating complementary and alternative medicine. In: Clinical Research in Complementary Therapies: Principles, Problems and Solutions. Edinburgh: Churchill Livingston; 2003:29-43.

13. Greenhalgh T. Searching the literature. In: How to Read a Paper; The Basics of Evidence-Based Medicine. London: Wiley Blackwell; 2010:15-28.

14. Craig P, Dieppe P, Macintyre S, Michie S, Nazareth I, Petticrew M. Developing and evaluating complex interventions: new guidance. Medical Research Council, 2008. Online document at http://www.mrc.ac.uk/Utilities/Document record/index.htm?d = MRC004871 accessed November 01, 2011.

15. Cochrane Collaboration. Unit Eight: Principles of Criticial Appraisal. Online document at http://ph.cochrane.org/ sites/ph.cochrane.org/files/uploads/Unit Eight.pdf Accessed October 10, 2012.

16. Cochrane Collaboration. Chapter 8: Assessing risk of bias in included studies. Online document at http://hiv.cochrane .org/sites/hiv.cochrane.org/files/uploads/Ch08_Bias.pdf Accessed June 02, 2011.

17. Gunnarsdottir TJ, Jonsdottir H. Does the experimental design capture the effects of complementary therapy? A study using reflexology for patients undergoing coronary artery bypass graft surgery. J Clin Nurs 2007;16:777-785.

18. Frankel BSM. The effect of reflexology on baroreceptor reflex sensitivity, blood pressure and sinus arrhythmia. Complement Ther Med 1997;5:80-84.

19. Hodgson NA, Andersen S. The clinical efficacy of reflexology in nursing home residents with dementia. J Altern Complement Med 2008;14:269-275.

20. Zhen LP, Fatimah SN, Acharya RU, Tam D-WD, Joseph KP. Study of heart rate variability due to reflexological stimulation. Clin Acupuncture Orient Med 2004;4:173-178.

21. Wilkinson ISA, Prigmore S, Rayner CF. A randomisedcontrolled trail examining the effects of reflexology of patients with chronic obstructive pulmonary disease (COPD). Complement Ther Clin Pract 2006;12:141-147.

22. Joseph P, Acharya IR, Kok Poo C, et al. Effect of reflexological stimulation on heart rate variability. Innovation et technologie en biologie et medecine 2004;25:40-45.

23. Mackereth PA, Booth K, Hillier VF, Caress AL. Reflexology and progressive muscle relaxation training for people with multiple sclerosis: A crossover trial. Complement Ther Clin Pract 2009;15:14-21. 
24. McVicar AJ, Greenwood CR, Fewell F, D'Arcy V, Chandrasekharan S, Alldridge LC. Evaluation of anxiety, salivary cortisol and melatonin secretion following reflexology treatment: A pilot study in healthy individuals. Complement Ther Clin Pract 2007;13:137-145.

25. Sudemeier H, Bodner G, Egger I, Mur E, Ulmer H, Herold $\mathrm{M}$. Changes of renal blood flow during organ-associated foot reflexology measured by color Doppler sonography. Forsch Komplementarmed 1999;6:129-134. (In German.)

26. Mur E, Schmidseder S, Egger I, et al. Influence of Reflex Zone Therapy of the Feet on Intestinal Blood Flow Measured By Color Doppler Sonography. Forsch Komplementarmed Klass Naturheilkd 2001;8:86-89. (In German.)

27. Hayes J, Cox C. Immediate effects of a five-minute foot massage on patients in critical care. Intensive Crit Care Nurs. 1999:15;77-82.

28. Hatton J, King L, Griffiths P. The impact of foot massage and guided relaxation following cardiac surgery: a randomised controlled trial. J Adv Nurs 2002;37:199-207.

29. Bauer BA, Cutshall SM, Wentworth LJ, et al. Effect of massage therapy on pain, anxiety, and tension after cardiac surgery: A randomized study. Complement Ther Clin Pract 2010;16:70-75.

30. Ejindu A. The effects of foot and facial massage on sleep induction, blood pressure, pulse and respiratory rate: Crossover pilot study. Complement Ther Clin Pract 2007;13:266-275.

31. Aourell M, Skoog M, Carleson J. Effects of Swedish massage on blood pressure. Complement Ther Clin Pract 2005;11: 242-246.

32. Critical Appraisal Skills Programme. Making sense of evidence: 10 questions to help you make sense of randomized controlled trials. Online document at http://calder.med .miami.edu/portals/ebmfiles/UM\%20CASP\%20RCTs\%20 Assessment\%20Tool.pdf accessed August 15, 2010.

33. Machi Y, Lui C, Fujita M. Physiological measurements for reflexology foot massage. J Int Soc Life Info Sci 2000;18:502-507.

34. Zhang W, Takahashi S, Miki T, Fujieda H, Ishida T. A pilot study exploring the effects of reflexology on cold intolerance. J Acupunct Meridian Stud. 2010;3:43-48.

35. Byers DC. Better Health With Foot Reflexology, 2nd ed. St. Petersburg, FL: Ingham Publishing Inc. 1994.

36. Moher D, Liberati A, Tetzlaff J, Altman DF, PRISMA Group. Preferred reporting items for systematic reviews and meta analyses: The PRISMA Statement. PLoS Med. 6(7):e1000097. doi:10.1371/journal.pmed.1000097

37. Oleson T, Flocco W. Randomized controlled study of premenstrual symptoms treated with ear, hand, and foot reflexology. Obstet Gynecol 1993;82:906-911.

38. Brygge $\mathrm{T}$, Heinig $\mathrm{JH}$, Collins $\mathrm{P}$, et al. Reflexology and bronchial asthma. Respir Med 2001;95:173-179.
39. Williamson J, White A, Hart A, Ernst E. Randomised controlled trial of reflexology for menopausal symptoms. BJOG 2002;109:1050-1055.

40. Ross SK, Hamilton J, Macrae G, Docherty C, Gould A, Cornbleet MA. A pilot study to evaluate the effect of reflexology on mood and symptom rating of advanced cancer patients. Palliat Med 2002;16:544-545.

41. Tovey P. A single-blind trial of reflexology for irritable bowel syndrome. Br J Gen Pract 2002;52:19-23.

42. Siev-Ner I, Gamus D, Lerner-Geva L, Achiron A. Reflexology treatment relieves symptoms of multiple sclerosis: A randomized controlled study. Mult Scler 2003;9:356-361.

43. Quinn F, Hughes CM, Baxter GD. Reflexology in the management of low back pain: A pilot randomised controlled trial. Complement Ther Med 2008;16:3-8.

44. Ho-Leung JM, Willy CC, To W, Yu Sun JL, Wai Mei AT. Randomized controlled trial of foot reflexology for patients with symptomatic idiopathic detrusor overactivity. Int Urogynecol J 2007;18:653-658.

45. Holt J, Lord J, Acharya U, et al. The effectiveness of foot reflexology in inducing ovulation: a sham controlled randomised trial. Fertil Steril 2009;91:2514-2519.

46. Heather H. Does reflexology impact on cancer patients' quality of life? Nurs Stand 2000;14(31):33-38.

47. Jones J, Thomson P, Lauder W, Leslie SJ. Reported treatment strategies for reflexology in cardiac patients and inconsistencies in the location of the heart reflex point: an online survey. Complementary therapies in clinical practice. 2012,18(3);145-150, Epub 2012 Jun 27.

48. Mackereth PA, Tiran D. Reviewing theories and origins. In: Clinical Reflexology: A Guide for Health Professionals. Edinburgh: Churchill Livingstone; 2002:5-17.

49. Jones J, Thomson P, Lauder W, Leslie SJ. A proposed reductionist solution to address the methodological challenges of inconsistent reflexology maps and poor experimental controls in reflexology research: A disussion paper. J Altern Complement Med [in press]. Accepted March 2012.

Address correspondence to: Jenny Jones, BSc (Hons), RN School of Nursing, Midwifery \& Health University of Stirling Highland Campus Old Perth Road Inverness, IV2 3JH United Kingdom

E-mail: jenny.jones@stir.ac.uk 